\title{
Visual function after bilateral implantation of multifocal versus monofocal IOLs in children below 5 years of age
}

\author{
Marek E. Prost \\ Department of Ophthalmology, Military Institute of Aviation Medicine \\ Acting Head: Jaromir Wasyluk, MD, PhD \\ Center for Pediatric Ophthalmology \\ Head: Ewa Oleszczyńska-Prost, MD, PhD
}

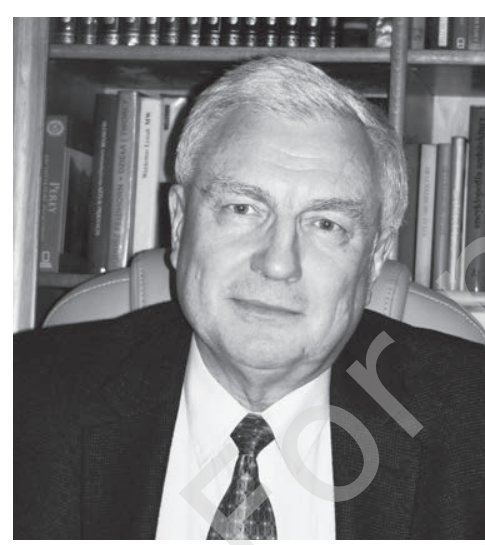

H I G H LI GHTS

Implantation of a multifocal IOL in small children provides a better level of near vision and considerably improves the development of stereopsis in comparison with children after monofocal IOL implantation.

\section{ABSTRACT}

Background: Since pediatric ophthalmologists still prefer to implant monofocal IOL and experience with multifocal IOL implantation in children is limited. Therefore, the aim of the study was to evaluate visual results after bilateral implantation of multifocal versus monofocal IOLs in children aged 1-5 years with congenital cataract.

Methods: Children between 1 and 5 years of age with bilateral cataract who underwent cataract removal implantation of an multifocal IOL - Lentis Mplus $^{\mathrm{x}}$, Oculentis GmbH, Germany (55 children - group A) or monofocal IOL - C-flex Aspheric Monofocal, Rayner Intraocular Lenses Limited, UK (55 children - group B). The distance corrected visual acuity, distance corrected near visual acuity, binocular function using the Worth 4-dot test, stereopsis using the TNO test were evaluated.

Results: At the final follow-up visit after 12 months, the mean best corrected distant visual acuity was not significantly different between groups A and B but the best corrected near visual acuity, binocular vision and stereopsis were significantly better in patients from group A as compared to group B.

Conclusion: Implantation of a multifocal IOL in children aged 1-5 years with bilateral cataract provides a better level of near vision and considerably improves the development of stereopsis in comparison with children after monofocal IOL implantation.

Key words: pediatric cataract surgery, multifocal IOLs, monofocal IOLs, distance and near visual acuity, binocular vision, stereopsis 


\section{INTRODUCTION}

Implantation of multifocal IOLs is nowadays widely used method of aphakia correction after cataract surgery in adult patients $[1,2]$. A new generation of multifocal IOLs has demonstrated their safety and efficacy $[3,4]$. In pediatric patients implantation of IOLs is now accepted as an optimal method of visual rehabilitation after cataract surgery. However, pediatric ophthalmologists prefer to implant monofocal IOL in children. These IOLs provide excellent distance vision correction but additional reading spectacles with monofocal or bifocal glasses are required for near vision. It is known that loss of accommodation has a great effect on the development of visual function in pediatric patients [5-7]. It considerably affects the development of binocularity and stereopsis in children and might worsen amblyopia (especially in unilateral pseudophakia) [5]. The visual world of our youngest patients is primarily at our fingertips, so it is important to give a child a focused image of a toy, bottle, or a mother's face. Therefore, the implantation of multifocal IOLs should theoretically offer much better conditions for the development of visual functions in children. However, multifocal IOLs still have some drawbacks: considerable loss of light (diminished contrast sensitivity), glare and halos, disturbed night vision, simultaneous vision of 2 images on the retina (diffractive IOLs), small visual fields of distant and near segments and a necessity for continuous searching of distant or near picture (refractive IOLs). It is still unknown how multifocality (and its drawbacks) might influence the development of binocular vision. Due to all these problems pediatric ophthalmologists still prefer to implant monofocal IOLs and our experience with multifocal IOL implantation in children is very limited. In the literature one can find only a few papers concerning this problem [8-11] and authors encourage surgeons who have implanted multifocal IOLs in children to report their experience so as to increase the number of cases reported and hence improve the discussion on the possible benefits and drawbacks of this type of surgery [9].

In recent years a new concept of multifocal IOL was developed - multifocal, asymmetric, refractive IOL (Lentis Mplus, Oculentis GmbH, Germany). This IOL is one-piece zonal refractive lens with plate haptics and two refractive segments - a larger, upper and central zone for distance and smaller, lower segment with addition +3.0 D for the near (fig. 1). Due to its optic design Lentis Mplus offers much better contrast sensitivity and much smaller light loss than rotationally symmetric IOLs, pupil indipendence and only one image focused at the same time on the retina. Accordingly, many drawbacks of previous multifocal IOLs, which deterred pediatric ophthalmologists from their pediatric implantation, have been corrected.

The aim of the study was to evaluate visual results after bilateral implantation of multifocal, asymmetric versus monofocal IOLs in children aged $1-5$ years.

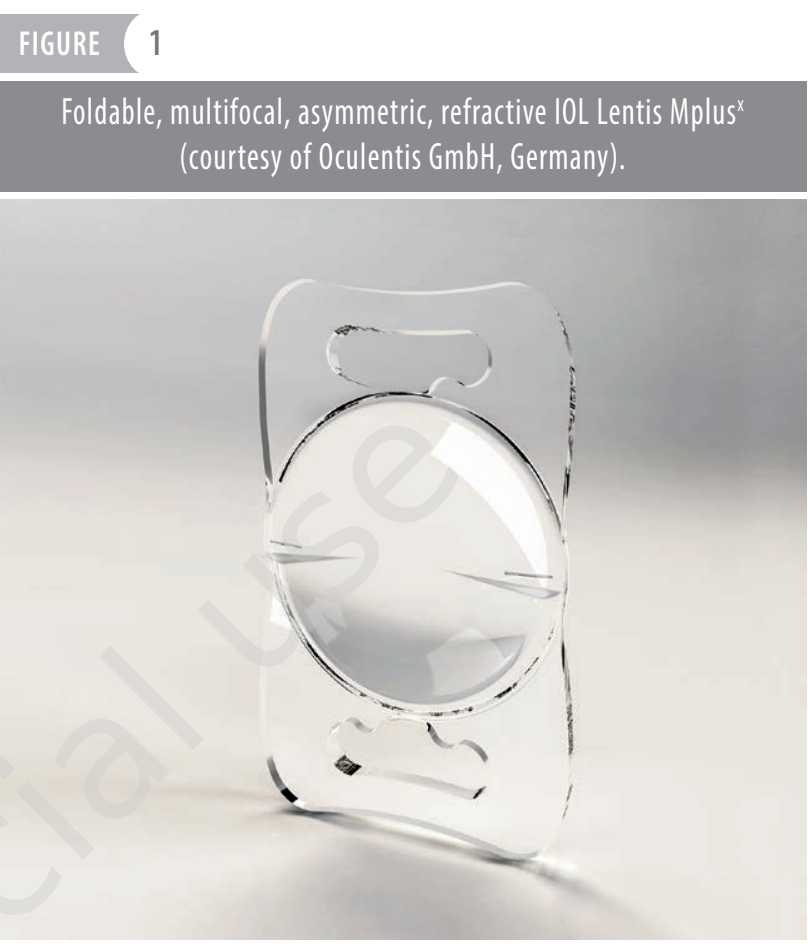

\section{PATIENTS AND METHODS}

\section{Patient selection}

A total of 110 children aged 1-5 years with bilateral congenital cataract were enrolled for the study in the Center for Pediatric Ophthalmology in Warsaw/Poland from May 2012 to November 2015. Indications for surgery included visual acuity 0.4 or worse (in older children) or dense central lens opacification larger than $3 \mathrm{~mm}$ and obscuring visual axis (in younger, illiterate children). The main exclusion criteria were: recurrent chronic uveitis, previous ocular trauma and existent or suspected corneal, iris, retinal or optic nerve pathologies. Patients were enrolled prospectively over a study period. The selection of multifocal or monofocal IOL was made after discussing it with children's parents. Then, the patients were divided into two groups group A implanted with multifocal IOL in both eyes and group B implanted with monofocal IOL in both eyes.

In each case parents informed consent was obtained and detailed information concerning advantages and disadvantages of each IOL were provided.

\section{Preoperative examination}

Before surgery, a complete ophthalmological and systemic examination was performed. It included Snellen's visual acuity assessment for distance (letters or numbers) for older children or Allen figure optotypes for younger children (Precision Vision chart no. 2503), near vision assessment using reading cards with cord for $40 \mathrm{~cm}$ (Precision Vision charts no. 2508 or no. 2709), intraocular pressure (IOP) 
measured by Perkins applanation tonometry, assessment of ocular deviations using synoptophore or PlusoptiX SO4 device (PlusoptiX GmbH, Germany), cycloplegic refraction assessment and keratometry (using handheld autorefractometer Retinomax 3 (Righton Co., Japan), portable slit-lamp biomicroscopy and fundus evaluation under dilation with binocular indirect ophthalmoscopy. Binocular function was evaluated with the Worth 4 dot test (Gulden Ophthalmics, USA) and near stereoacuity with TNO stereotest (Laméris Ootech BV, Netherlands). Worth test was performed at the $5 \mathrm{~m}$ distance with the use of handheld Worth 4 dot test at $5 \mathrm{~m}$ distance. With the TNO test, the booklet was held at $40 \mathrm{~cm}$ perpendicular to the subject's visual axis and the screening plates from 480 to 15 seconds of arc were gradually presented until the child was able to identify three-dimensional shape correctly.

Axial length and anterior chamber depth were measured using ultrasound technique (AL-4000, Tomey Corporation, Japan) and IOL power calculation was performed using Hoffer Q formula corrected to the age according to the recommendations of Trivedi and Wilson to minimize late myopia [12].

\section{Intraocular lens}

Foldable, multifocal, asymmetric, refractive IOLs (Lentis Mplus and Lentis Mplus ${ }^{\mathrm{x}}$ (since October 2013), Oculentis $\mathrm{GmbH}$, Germany) were implanted in both eyes of 55 children (group A) (fig. 1) and foldable monofocal IOLs (C-flex Aspheric Monofocal, Rayner Intraocular Lenses Limited, UK) were implanted in both eyes of 55 children (group B).

\section{Surgical technique}

All cataract surgeries were performed by a single experienced surgeon (M.E.P.) under general anesthesia. Standard surgical technique of C-flex Aspheric Monofocal IOLs included: limbal corneal approach, manual continuous curvilinear capsulorhexis, hydrodissection, phacoaspiration of the nuclear and cortical lens material, posterior capsulectomy of $4 \mathrm{~mm}$ and anterior vitrectomy through the main incision using vitrector, injection of an ophthalmic viscosurgical device (OVD) to open widely the capsular bag and IOL implantation in the capsular bag. After OVD removal, the main wound was closed with one 10.0 nylon suture.

In children with Lentis Mplus IOLs different technique was used. Lentis Mplus IOLs with plate design have different unfolding pattern during removal from the cartridge noozle which causes difficulties in precise placement of IOL in the peripheral part of the capsular bag after posterior capsulectomy and anterior vitrectomy. Therefore, in these children surgical technique included: limbal corneal approach, manual continuous curvilinear capsulorhexis, hydrodissection, phacoaspiration of the nuclear and cortical lens material, injection of an ophthalmic viscosurgical device, implanta- tion in the bag IOL and pars plana posterior capsulectomy of $4 \mathrm{~mm}$ and anterior vitrectomy using vitrector. After OVD removal, the main wound was closed with one 10.0 nylon suture and pars plana wound with one 8.0 absorbable suture (fig. 2).

\section{FIGURE ( 2}

Lentis Mplusx $10 \mathrm{~L}$ after implantation in 1.5 year old child.

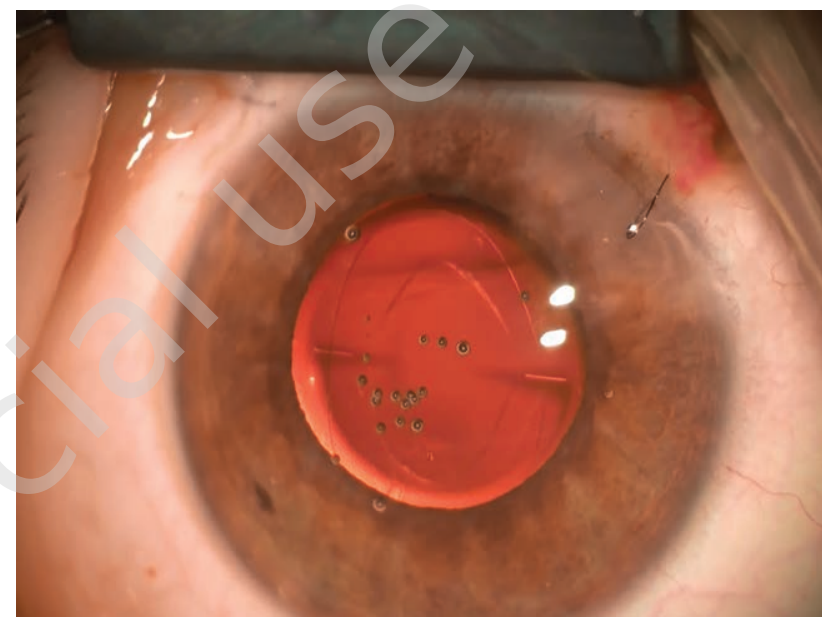

At the conclusion of surgery pupil was constricted by intracameral injection of acetylcholine, $1 \mathrm{~g}$ of cefuroxime was injected into the anterior chamber and a sub-conjunctival injection of $4 \mathrm{mg}$ of dexamethasone was given.

\section{Postoperative care}

Postoperatively, all the patients received topical moxifloxacin $0.5 \%$ four times a day for 7 days and loteprednol etabonate $0.5 \%$ five times a day for 2 weeks and titrated further over a period of next two weeks. A clear protector was worn for 2-3 weeks after surgery to avoid possible trauma. Postoperative examinations included distant and near visual acuity, IOP measurements, biomicroscopy of the anterior segment, eye fundus examination after pupil dilation, binocular vision, and stereoacuity measurements and assessment of ocular deviations (with the previously mentioned methods) performed at 3, 6, 9 and 12 months thereafter.

Preoperative and postoperative measurements of distant and near visual acuity, binocular vision and stereoacuity were made by the same orthoptist.

Occlusion therapy and binocular vision training was started in the postoperative period depending on the presence or absence of amblyopia and on the development of binocular vision.

Because children were only $1-5$ years old, it was impossible to evaluate the occurrence of glare and halos but their parents were asked to ask them for the presence of these visual symptoms later than 3 months after the surgery. 


\section{Statistical analysis}

Mann-Whitney U test for non-parametric data was used for comparison of best corrected distant visual acuity, best corrected near visual acuity an results of TNO tests and chi-squared test $\mathrm{x}^{2}$ test for Worth 4 dot test.

\section{RESULTS}

\section{Patients}

Mean age of children at the time of surgery was 2.3 years (range 1,1-5 years) in group A and 2.6 years (range 1,3-5 years) in group $B$.

\section{Surgical results}

We reported no intraoperative or postoperative complications in all operated children. In one child with implanted Lentis Mplus IOL it was necessary to perform surgical capsulectomy because of LEC proliferation and phimosis of the primary posterior capsulectomy. Visual axis of all other children was clear during the follow-up period.

\section{Distant visual acuity}

Before the surgery, it was possible to measure distant visual acuity in 36 children in group A and in 37 children in group B. After the surgery, the measurements were performed in 38 children in group A and in 39 children in group B.

The best corrected distant visual acuity was not significantly different between group A (0.48) and group B (0.41) one year postoperatively $(\mathrm{p}=0.565$, Mann-Whitney $U$ test) (tab. 1).

\section{Near visual acuity}

Before the surgery, it was possible to measure near visual acuity in 37 children in group A and in 38 children in group B. After the surgery, the measurements were performed in 40 children in group A and in 41 in group B.

The best corrected near visual acuity was significantly superior in patients in group A (0.70) as compared to group $\mathrm{B}(0.44)$ one year postoperatively ( $\mathrm{p}<0.02$, Mann-Whitney U test) (tab. 2).

\section{Worth test}

Before the surgery, it was possible to perform Worth test in 37 children in group A and in 39 children in group B. After the surgery, the measurements were performed in 40 children in group A and in 42 children in group B.

Binocular function evaluated with the Worth 4-dot test was significantly superior in patients from group A (positive in $46 \%$ ) as compared to group B (positive in $21 \%$ ) one year postoperatively (chi-squared test $\mathrm{x}^{2}$ test $=4.8, \mathrm{df}=1$, $\mathrm{p}=0.028)$ (tab. 3).

\section{TNO test}

Before the surgery, it was possible to measure stereoacuity for near in 34 children in group A and in 33 children in group B. After the surgery, the measurements were performed in 37 children in group A and 35 in group B (tab. 4). Near stereoacuity was significantly better in patients in group A (220 arcsec) as compared to group B (380 arcsec) one year postoperatively ( $\mathrm{p}<0.002$, Mann-Whitney U test).

\section{The occurrence of glare and halos}

The parents of both operated children did not report the occurrence of glare and halos later than 3 months after the surgery.

\section{TABLE $(1$}

Best corrected distant visual acuity before and 1 year after surgery.

\begin{tabular}{c|c|c} 
& Group A (bifocal IOL) & Group B (monofocal IOL) \\
\hline BEFORE SURGERY & 0.1 & 0.15 \\
(range $0.01-0.2)$ & (range $0.02-0.3$ ) \\
\hline 1 YEAR AFTER SURGERY & 0.48 & 0.41 \\
(range $0.04-0.8)$ & (range $0.05-0.5)$
\end{tabular}

\section{TABLE $(2$}

\section{Best corrected near visual acuity before and 1 year after surgery.}

\begin{tabular}{|c|c|c|}
\hline & $\begin{array}{l}\text { Group A (bifocal IOL) } \\
\text { (with distant correction) }\end{array}$ & $\begin{array}{c}\text { Group B (monofocal IOL) } \\
\text { (with addition for near) }\end{array}$ \\
\hline BEFORE SURGERY & $\begin{array}{c}0.15 \\
\text { (range } 0.01-0.1)\end{array}$ & $\begin{array}{c}0.18 \\
\text { (range } 0.02-0.3)\end{array}$ \\
\hline 1 YEAR AFTER SURGERY & $\begin{array}{c}0.70 \\
\text { (range } 0.2-1.0)\end{array}$ & $\begin{array}{c}0.44 \\
\text { (range } 0.1-0.6)\end{array}$ \\
\hline
\end{tabular}




\begin{tabular}{c|c|c}
\hline & \multicolumn{2}{|c}{ Results of Worth test before and 1 year after surgery. } \\
\hline BEFORE SURGERY & $\begin{array}{c}\text { Group A (bifocal IOL) } \\
\text { (with distant correction) }\end{array}$ & $\begin{array}{c}\text { Group B (monofocal IOL) } \\
\text { (with addition for near) }\end{array}$ \\
\hline 1 YEAR AFTER SURGERY & - & - \\
\hline
\end{tabular}

\begin{tabular}{c|c|c}
\hline TABLE 4 & \multicolumn{2}{|c}{ Results of TN0 test before and 1 year after surgery. } \\
\hline BEFORE SURGERY & $\begin{array}{c}\text { Group A (bifocal IOL) } \\
\text { (with distant correction) }\end{array}$ & $\begin{array}{c}\text { Group B (monofocal IOL) } \\
\text { (with addition for near) }\end{array}$ \\
\hline 1 YEAR AFTER SURGERY & negative in all examined patients & negative in all examined patients \\
\hline
\end{tabular}

\section{Discussion}

In the literature one can find only a few papers concerning the results of implantations of multifocal IOLs in children [8-11]. All these papers report encouraging results. However, in some of them both unilateral and bilateral cases were included in the study $[8,11]$ and only a small group of five children with unilateral cataracts was evaluated [9]. The only report comparing homogenous large enough groups of pediatric patients was paper by Ram et al. who evaluated visual results and complications after bilateral implantation of multifocal versus monofocal IOLs in 21 children [10]. In all these papers different multifocal IOLs were implanted, but all of them have similar drawbacks (as mentioned above) which deter most pediatric ophthalmologists from implanting them in children. Several years ago a new multifocal IOL - multifocal, asymmetric, refractive IOL (Lentis Mplus, Oculentis GmbH, Germany) was introduced. This IOL has low loss of light of about $7 \%$ as compared to other refractive and diffractive IOLs which have light loss of 14-22\% (at the beginning of the study) [13]. Contrast perception with the Lentis Mplus is equivalent to that of a 20-year-old with healthy eyes [14]. The IOL is pupil indipendent and only one image is focused at the same time on the retina. Recently, Oculentis has introduced a new IOL - Lentis Mplus ${ }^{\mathrm{x}}$ with improved optics which simplifies neuronal image interpretation by the retinal rods and cones due to additive paraxial asphericity and surface design optimization which has enlarged the near vision section making the IOL more pupil independent and providing better reading conditions. According to the manufacturer, this IOL has a very low loss of light of only 5\% [15]. Therefore, the author, after many years of considerations whether to implant multifocal IOLs in children, decided to use this IOL in pediatric children.
The implantation technique of Lentis Mplus IOLs is more difficult than of other multi- or monofocal IOLs in children. Posterior capsulectomy and anterior vitrectomy are commonly performed through the main incision using vitrector and then IOL is implanted in the bag with the help of spatula which directs the lower haptics into the remaining peripheral bag. Lentis Mplus IOLs have plate design and different, more vigorous unfolding pattern with marked IOL bending into the vitreous cavity when upper haptic is released from the cartridge noozle. It causes difficulties in precise placement of IOL in the peripheral parts of the capsular bag after posterior capsulectomy and anterior vitrectomy. Therefore, the author has changed the technique of IOL implantation performing posterior capsulectomy and anterior vitrectomy through pars plana after implanting IOL in the bag. Lentis Mplus IOL has asymmetric optic so it must be very precisely positioned in the bag. It has also good stability in the bag so its dialing is difficult as compared with other IOLs.

Examination of visual functions one year after IOL implantation have shown that the best corrected distant visual acuity was not significantly different between groups A and B. However, children with implanted multifocal IOLs have significantly better near vision and, most of all, binocular vision and stereopsis (Worth and TNO tests). The same tendency was observed in other studies in children with implanted symmetric multifocal IOLs [8-11]. Moreover, in adult patients multifocality does not deteriorate stereoacuity after the surgery [16]. We do not know the exact cause of better development of these visual functions in children with multifocal IOLs. However, this can be explained by the more prolonged use of near vision by these children. Children with monofocal IOLs require second eyeglasses or bifocal eyeglasses for near-vision activities. Both solutions 
are troublesome for the patients and their parents: glasses with monofocal lenses require frequent changes and bifocal eyeglasses are not widely accepted by children because of their appearance. Therefore, focused near vision activity of these children is limited (long periods of defocused near vision). Because the visual world of youngest patients is concentrated primarily on near distance, the development of visual functions in children should be less effective. Bilateral implantation of multifocal IOLs increases the time of focused near vision (it can be obtained by wearing monofocal eyeglasses only) and offers much better conditions for the development of near vision, binocular vision and stereopsis.

The author has found no publications concerning implantation of multifocal, asymmetric, refractive IOLs in children. However, the analysis of optical properties of multifocal, asymmetric, or refractive IOLs (better contrast sensitivity and small light loss, pupil indipendency and only one image focused at the same time on the retina) make them more suitable for the implantation in children than rotationally symmetric multifocal IOLs. The results of this study indicate that the implantation of multifocal, asymmetric, refractive IOLs offers better conditions for the development of visual functions in children after cataract surgery than monofocal IOLs. Therefore, the author have chosen multifocal, asymmetric, refractive IOLs as the standard IOLs for the implantation in pediatric cataract surgery.

\section{CONCLUSION}

Implantation of multifocal, asymmetric, refractive IOLs in small children aged 1-5 years with bilateral cataract provide better near vision and considerable improves the development of binocular vision and stereopsis. Although it was impossible to evaluate it directly, reports of the parents of these children indicate that photic phenomena such as glare and halos are not a problem after implantation of multifocal IOLs.

\section{References}

1. Leyland M, Zinicola E. Multifocal versus monofocal intraocular lenses in cataract surgery: a systematic review. Ophthalmology 2003; 110: $1789-1798$.

2. Calladine D, Evans JR, Shah S, et al. Multifocal versus monofocal intraocular lenses after cataract extraction. Cochrane Database of Systematic Reviews 2012; 9. Art. No.: CD003169. DOI: 10.1002/14651858.CD003169.pub3.

3. Souza CE, Muccioli C, Soriano ES, et al. Visual performance of AcrySof ReSTOR apodized diffractive IOL: a prospective comparative trial. Am J Ophthalmol 2006; 141: 827-832.

4. Braga-Mele R, Chang D, Dewey S, et al.; for the ASCRS Cataract Clinical Committee. Multifocal Intraocular Lenses: Relative indications and contraindications for implantation. J Cataract Refract Surg 2014; 40: 313-322.

5. Rychwalski PJ. Multifocal IOL implantation in children: Is the future clear? J Cataract Refract Surg 2010; 36: $2019-2021$.

6. Wright KW, Matsumoto E, Edelman PM. Binocular fusion and stereopsis associated with early surgery for monocular congenital cataracts. Arch Ophthalmol 1992; 110: 1607-1609.

7. Brown SM, Archer S, Del Monte MA. Stereopsis and binocular vision after surgery for unilateral infantile cataract. J AAPOS 1999; 3 : 109-113.

8. Jacobi PC, Dietlein TS, Konen W. Multifocal intraocular lens implantation in pediatric cataract surgery. Ophthalmology 2001; 108: 1375-1380.

9. Cristóbal J, Remoón L, Ángeles Del Buey M, Monstés-Micó R. Multifocal intraocular lenses for unilateral cataract in children. J Cataract Refract Surg 2010; 36: 2035-2040.

10. Ram J, Agarwal A, Kumar J, Gupta A. Bilateral implantation of multifocal versus monofocal intraocular lens in children above 5 years of age. Graefes Arch Clin Exp Ophthalmol 2014; 252: 441-447.

11. Abouzeid H, Moetteli L, Munier F. New-generation multifocal intraocular lens for pediatric cataract. Ophthalmologica 2013; 200: 100-107.

12. Trivedi RH, Wilson ME Jr. Intraocular lens power selection for children. In: Garg A, Lin JT, Latkany R, Bovet J, Haigis W (eds). Mastering the techniques of IOL power calculations. Jaypee Brothers 2005: 127-135.

13. Morris R. Mplus innovative multifocal IOL with unique optics. Presented at 17th ESCRS Winter Meeting, Warsaw 2013.

14. Auffarth GU. Lentis Mplus: An innovative multifocal lens technology. Cataract and Refractive Surgery Today Europe 2010; 2: 34-35.

15. Wanders B. Lentis Mplusx Get off the peaks, get into the zone. Cataract and Refractive Surgery Today Europe 2014; 2 Suppl: 3-4.

16. Ferrer-Blasco T, Madrid-Costa D, Garcia-Lázaro S, et al. Stereopsis in bilaterally multifocal pseudophakic patients. Graefes Arch Clin Exp Ophthalmol 2011; 249: 245-251. 